\title{
Effect of adherence on daytime sleepiness, fatigue, depression and sleep quality in the obstructive sleep apnea/hypopnea syndrome patients undertaking nasal continuous positive airway pressure therapy
}

\author{
This article was published in the following Dove Press journal: \\ Patient Preference and Adherence \\ 12 April 2017 \\ Number of times this article has been viewed
}

\section{Yeying Wang ${ }^{1,2}$ \\ $\mathrm{Li} \mathrm{Ai}^{\prime}$ \\ Jiahong Luo ${ }^{2}$ \\ Ran $\mathrm{Li}^{\prime}$ \\ Yanlin Chai' \\ Xiaojie $\mathrm{He}^{\prime}$ \\ Yu Cao' \\ Yongxia $\mathrm{Li}^{\prime}$}

'Department of Respiratory Medicine,

The Second Affiliated Hospital

of Kunming Medical University,

${ }^{2}$ Department of Epidemiology

and Biostatistics, School of

Public Health, Kunming Medical

University, Chenggong New City,

Kunming, Yunnan Province, People's

Republic of China
Correspondence: Yongxia Li Department of Respiratory Medicine, The Second Affiliated Hospital of Kunming Medical University, No 374 Dianmian Road, Kunming 65010I, Yunnan Province, People's Republic of China

Tel +86 I36 588I 0805

Fax +86 87I 65352087

Email liyongxia@hotmail.com
Objectives: The aims of this study were to describe changes in day- and nighttime symptoms and the adherence to nasal continuous positive airway pressure (nCPAP) during the first 3-month nCPAP therapy among newly diagnosed patients with obstructive sleep apnea/hypopnea syndrome (OSAS) and to identify the effect of adherence on the changes in day- and nighttime symptoms during the first 3 months.

Methods: Newly diagnosed OSAS patients were consecutively recruited from March to August 2013. Baseline clinical information and measures of the Epworth Sleepiness Scale (ESS), Fatigue Severity Scale (FSS), Zung's Self-Rating Depression Scale (SDS) and the Pittsburgh Sleep Quality Index (PSQI) at baseline and the end of 3rd, 6th, 9th and 12th week of therapy were collected. Twelve weeks' adherence was calculated as the average of each 3-week period. Mixed model was used to explore the effect of adherence to nCPAP therapy on ESS, FSS, SDS and PSQI in each 3-week phase.

Results: Seventy-six patients completed the 12-week follow-up. The mixed-effects models showed that under the control of therapy phase adherence in the range of $<4$ hours per night, using nCPAP could independently improve daytime sleepiness, in terms of ESS (coefficient, [95\% confidence interval] unit; $-4.49[-5.62,-3.36])$. Adherence at $4-6$ hours per night could independently improve all variables of day- and nighttime symptoms included in this study, namely ESS -6.69 (-7.40, -5.99), FSS -6.02 (-7.14, -4.91), SDS -2.40 (-2.95, -1.85) and PSQI $-0.20(-0.52,-0.12)$. Further improvement in symptoms could be achieved at $\geq 6$ hours per night using nCPAP, which was ESS -8.35 (-9.26, -7.44), FSS -10.30 (-11.78, -8.83), SDS $-4.42(-5.15,-3.68)$ and PSQI $-0.40(-0.82,-0.02)$. The interaction between adherence level and therapy phase was not significant in day- and nighttime symptoms.

Conclusion: The effect of adherence on the above-mentioned symptoms is stable through the first 3 months. Under the control of therapy phase, the nCPAP therapy effectively improves day- and nighttime symptoms with $\geq 4$ hours adherence, and the patients can achieve a further improvement with $\geq 6$ hours adherence.

Keywords: OSAS, nCPAP, Epworth Sleepiness Scale, Fatigue Severity Scale, Zung's SelfRating Depression Scale, the Pittsburgh Sleep Quality Index

\section{Background}

Obstructive sleep apnea/hypopnea syndrome (OSAS) is characterized by repetitive obstruction of the upper airway during sleep, causing obstructive apneas leading to 
oxygen desaturations and fragmentation of nocturnal sleep. ${ }^{1,2}$ Snoring, excessive daytime sleepiness (EDS), fatigue and depression are frequently associated. The more serious consequences of OSAS are higher risk of cardiovascular, pulmonary, vascular and cerebral diseases in patients with OSAS.

Continuous positive airway pressure (CPAP) therapy has been proven to significantly reduce the risk of cardiovascular disease as well as improve daytime symptoms. Effective CPAP management reduces nighttime breathing disturbances and improves oxygenation, sleep architecture, daytime sleepiness, daytime performance and depression. ${ }^{3-8}$ To achieve the effectiveness of CPAP therapy, OSAS patients are suggested to use CPAP during whole nights.

However, many patients could not follow this suggestion. Several previous studies have focused on the adherence to CPAP among OSAS patients. ${ }^{9-15}$ Some of the studies aimed to reveal the relationship between CPAP using hours and its effects on associated symptoms. ${ }^{10,11,16}$ Weaver et $\mathrm{al}^{11}$ reported that after a 3-month therapy, a linear dose-response trend is observed between the increase in CPAP using hours and the cumulative proportion of participants obtaining normal values of Epworth Sleepiness Scale (ESS $\leq 10$ is considered normal value), and they also found that 4 hours duration of using CPAP during the night was a cutoff point for normalized ESS score. A similar result was also reported by Antic et al. ${ }^{10}$ They found that of the OSAS patients using CPAP for $>7$ hours, $80.6 \%$ had a normal ESS score, and of those using CPAP between 2 and 4 hours, there were $52.4 \%$ of them with normalized ESS score after 3-month therapy. Compared with the major daytime symptom, sleepiness, the relationship between adherence and normalization of fatigue and depression was rarely reported. Wang et $\mathrm{al}^{9}$ reported that fatigue, depression and sleep quality were independent influencing factors of adherence. Conversely, the effect of adherence on fatigue and depression might impact the changes in symptoms as well as the subsequent adherence. Therefore, it is necessary to conduct a study to identify the effect of adherence not only on daytime sleepiness but also on fatigue, depression and sleep quality.

Furthermore, previous studies reported the effect of adherence to CPAP therapy on related clinical variables; these studies were of a before-after design and could not reveal the independent effect of adherence under the control of therapy phase on the changes in the variables over time or the interaction between adherence and therapy phase. Knowledge of the time frame for these clinical variables would be useful in the early evaluation of CPAP therapy.

\section{Methods Study sample}

According to the guideline of OSAS diagnosis and therapy, ${ }^{17}$ newly diagnosed OSAS patients who met the criteria of CPAP therapy (apnea/hypopnea index [AHI] $\geq 15$ or AHI $<15$ with complications) were consecutively recruited from March to August 2013 at the Sleep Center of The First People's Hospital of Yunnan Province and the Department of Respiratory Medicine of The Second Affiliated Hospital of Kunming Medical University. The patients with severe diseases or the patients who did not start nasal continuous positive airway pressure (nCPAP) therapy within 30 days after titration trial at the sleep center were excluded.

\section{Sleep test}

The diagnostic polysomnography (Alice 5; Respironics, Murrysville, PA, USA) included electroencephalography, electrooculography, chin electromyography, airflow at the nose and mouth, abdominal and chest movements, electrocardiography, sleep position and snoring frequency. An apneic episode was defined as a complete cessation of airflow or reduction in airflow by $>90 \%$ of the baseline for at least 10 seconds; a hypopneic episode was defined as a reduction in airflow by $>30 \%$ of the baseline for at least 10 seconds in association with a fall in arterial oxygen saturation of at least $4 \%$ or $50 \%$ of the baseline for at least 10 seconds in association with a fall in arterial oxygen saturation of at least $3 \% .{ }^{17}$ A titration trail would be conducted after sleep study, if the person met the criteria of taking CPAP therapy. ${ }^{17}$

\section{Data collection}

Demographic data, height, weight, circumferences of neck, waist and hip of each subject were recorded at the beginning and the end of the study at hospitals. Subjective daytime sleepiness, fatigue, depression and sleep quality were measured using the $\mathrm{ESS}^{18}$ (0-24, higher score indicated sleepier), Fatigue Severity Scale (FSS, 9-63, higher score indicated more fatigue), ${ }^{19}$ Zung's Self-Rating Depression Scale (SDS, 20-80, higher score indicated more depressed) ${ }^{20}$ and The Pittsburgh Sleep Quality Index (PSQI, 0-21, higher score indicated lower sleep quality $)^{21}$ before the onset of therapy and at the end of 3rd, 6th, 9th and 12th week of therapy from the date of starting nCPAP therapy. After the patient had started nCPAP therapy, the well-trained research team members made appropriate appointments and then interviewed these patients via telephone at the end of 3rd, 6th and 9th week of therapy. The first and last interviews took place at hospital before sleep studies. Use of nCPAP 
was recorded by a memory card in the nCPAP, which stored the daily information on the times at pressure. Subjects were recommended to bring the $\mathrm{nCPAP}$ to the clinics to download the records at the end of the 12 th week.

The protocol of this study was approved by the ethics committees of Prince of Songkla University and The Second Affiliated Hospital of Kunming Medical University. Written informed consent was obtained from all eligible participants.

\section{Data analysis}

ESS, FSS, SDS and PSQI scores were initially compared with 3-week-average nightly adherence hours using scatter plots and Spearman correlation coefficients. For convenience in subsequent analysis, average adherence was cut into 3 ranges: $<4$ hours, $4-6$ hours and $\geq 6$ hours. Four scores were compared across the 3 adherence ranges at each time using one-way analysis of variance (ANOVA) and $F$-test. Where the $F$-test was significant, pairwise comparisons were tested using two-sample $t$-test. Comparisons across therapy phases were made and tested by fitting mixed-effects randomintercept linear regression models in which adherence range and therapy phase and their interaction were fitted. This was necessary because the patient composition in any one adherence range changed from phase to phase.

As measurements were taken for each patient on 5 occasions (at baseline, 3rd, 6th, 9th and 12th week of therapy), the relationships of adherence, therapy phase and baseline variables with outcome parameters were modeled using mixed-effects randomintercept linear regression models in which the need for fitting an interaction between adherence range and therapy phase was explored. Mixed-effects regression was used to identify the effect of adherence level on ESS, FSS, SDS and PSQI. Adherence level, therapy phase and their interaction and other potential influencing factors were initially included in the models and considered to act as fixed-effect variables, whereas patients' common variable (the order of patient recruited in this study) was considered to be the random-effect variable. The covariance structure of mixed-effect model enables investigation of the assumption of equal variance for random effects. Manual backward exclusion method was used to refine the models, the variables without significant contribution to the fit of model being removed sequentially according to logrestricted maximum likelihood values. All the significance tests were 2 -sided, and $P$-values $<0.05$ were considered as indicating statistical significance.

\section{Results}

In this study, 80 newly diagnosed OSAS patients were recruited and 76 patients completed a 12-week follow-up. Among
76 patients, over three quarter of them were middle-aged males, and most of them were office workers. At the baseline, the mean values of body mass index (BMI) and waistand-hip-ratio were approximately $27 \mathrm{~kg} / \mathrm{m}^{2}$ and 1 , respectively (Table 1). The overall mean of the average duration of nightly use of nCPAP during 12 weeks was $5.60 \pm 1.99$ hours.

Figure 1 shows the crude relationship between adherence and ESS and FSS scores in each 3-week period. After first 3-week therapy, the lower adherence was related to greater concurrent sleepiness and more fatigue. As shown in Figure 2, with the progress of therapy, the good adherence correlated with low SDS scores, meanwhile the poor adherence correlated with high SDS scores. Similar relationship was also observed for the PSQI scores (Figure 2). These relationships were evident from the second 3-week period onward.

Subsequent analysis was based on the adherence cut into 3 ranges: $<4$ hours, $4-6$ hours and $\geq 6$ hours. Table 2 shows the distributions of adherence levels in each of the fourth 3-week therapy. During the first 3 weeks of therapy, $\sim 8 \%$ of the patients used nCPAP for $<4$ hours per night (mean [SD], 3.57 [0.41]), while $\sim 17 \%$ of the patients used nCPAP $\geq 6$ hours per night (6.37 [0.20]), and three quarter patients used it 4-6 hours per night (5.44 [0.38]). However, the proportion of adherence of 4-6 hours per night decreased through subsequent therapy phases. This was demonstrated by an increase in patients using nCPAP $<4$ hours and an increase in patients using it for $\geq 6$ hours. After the first 3 -week therapy, over $50 \%$ of the patients in each 3 -week period used nCPAP $\geq 6$ hours.

Table 3 shows ESS, FSS, SDS and PSQI scores throughout the 3-month nCPAP therapy. During first therapy phase,

Table I Demographic and relative clinical information of 76 OSAS patients at baseline

\begin{tabular}{ll}
\hline Items & Value \\
\hline Age (years) & $48.12 \pm$ I4.46 \\
Sex & \\
Male & $58(76.3)$ \\
Female & $18(23.7)$ \\
Occupation & \\
Official & $39(51.3)$ \\
Businessmen & $17(22.4)$ \\
Freelance & $8(10.5)$ \\
Retired & $6(7.9)$ \\
Others & $6(7.9)$ \\
BMI (kg/m $\left.{ }^{2}\right)$ & $26.97 \pm 3.33$ \\
Waist-and-hip-ratio & $0.96 \pm 0.06$ \\
AHI (hour ${ }^{-1}$ ) & $51.24 \pm 27.24$ \\
\hline
\end{tabular}

Notes: Mean \pm standard deviation; $n$ (\%).

Abbreviations: OSAS, obstructive sleep apnea/hypopnea syndrome; BMI, body mass index; $\mathrm{AHI}$, apnea/hypopnea index. 

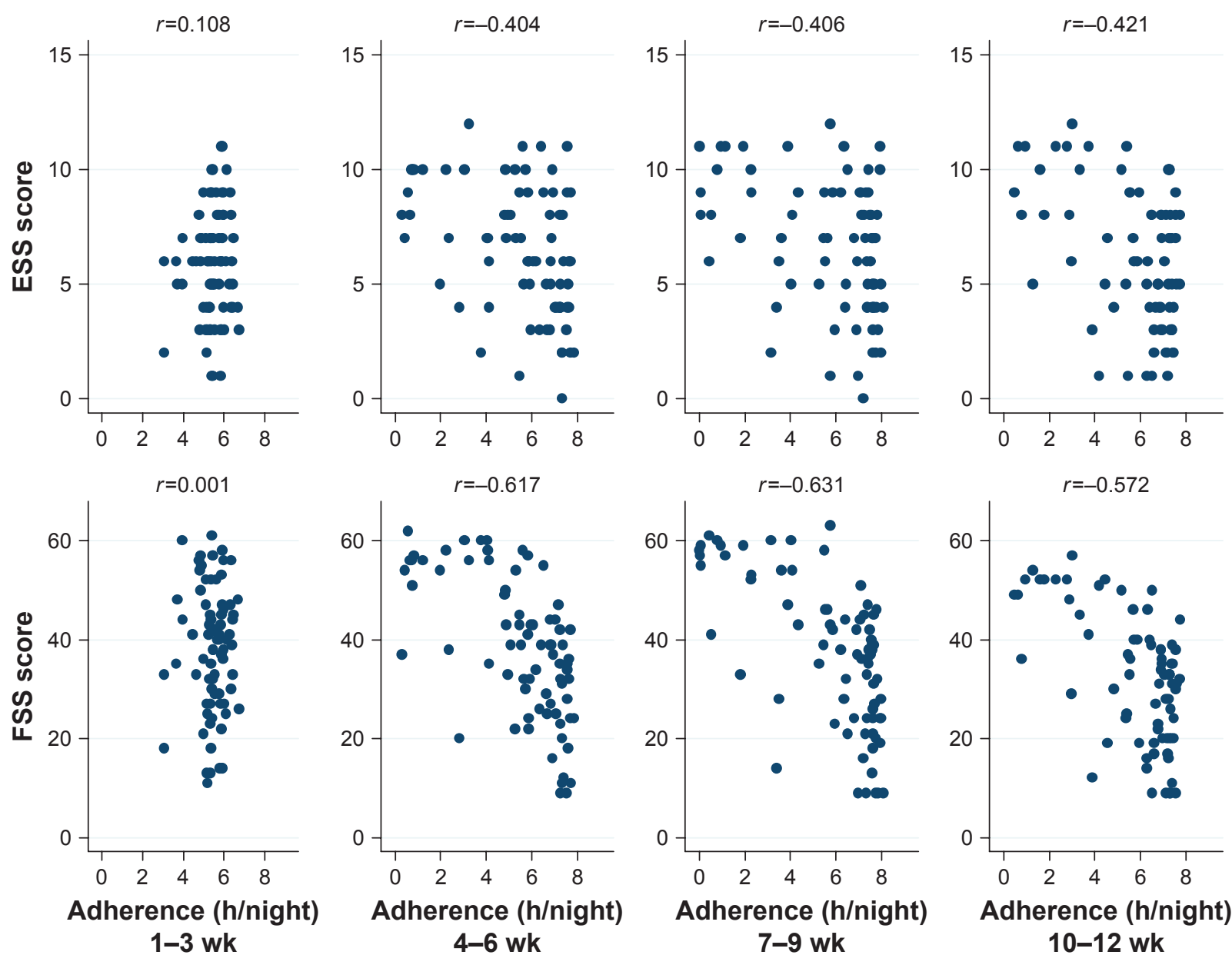

Figure I The crude relationship between adherence and ESS and FSS scores in each 3-week period.

Notes: Each point represents one study subject. ESS, Epworth Sleepiness Scale (0-24, higher score indicated sleepier); FSS, Fatigue Severity Scale (9-63, higher score indicated more fatigue).

Abbreviations: h, hour; wk, weeks.

ESS score rapidly reduced from a mean of 12.6 at baseline to between 5 and 6 in all adherence levels, and there were no significant differences among 3 adherence levels. However, with the progression of therapy, the adherence levels showed significantly different effects on ESS score. After first therapy phase, adherence $\geq 6$ hours level maintained the efficacy of first therapy phase, but $<4$ hours level was associated with increase in ESS score at the second therapy phase, which was maintained at 8-9. The lack of statistical significance in the ESS score at $<4$ hours level over the therapy phases was a result of the different patient compositions in this adherence range, with additional patients occupying this adherence range after the 3 rd week. However, the beneficial effect of increased adherence was evident from the second 3-week period onward (Table 3).

A similar pattern of FSS score and adherence was seen over the fourth 3-week periods. However, the FSS became significantly reduced over time in the $\geq 4$ hours adherence patients and was significant in the 4-6 adherence patients only at the last therapy phase, whereas the $\geq 6$ hours adherence patients showed a steady improvement throughout. Through the 4 therapy phases, the FSS scores of $<4$ hours adherence patients consistently had higher FSS scores than the patients with better adherence (Table 3).

The distributions of SDS score over the fourth 3-week period were consistent with FSS score. The SDS scores across 3 adherence ranges differed significantly from each other in each of the 4 therapy phases. The $\geq 6$ hours adherence patients had a steadily reduced SDS over time, while patients adhering for $<4$ hours or 4-6 hours group had a significantly reduced score only at the 10 - to 12 -week phase (Table 3).

After first 3-week therapy, PSQI scores significantly reduced in all 3 adherence ranges, and the reduced scores were maintained through to the end of the 12th week. However, from the 2nd phase on, the reduction was significantly greater with greater adherence hours (Table 3).

In multivariate modeling, the potential influence predictors on ESS, FSS, SDS and PSQI initially included in mixed-effects 

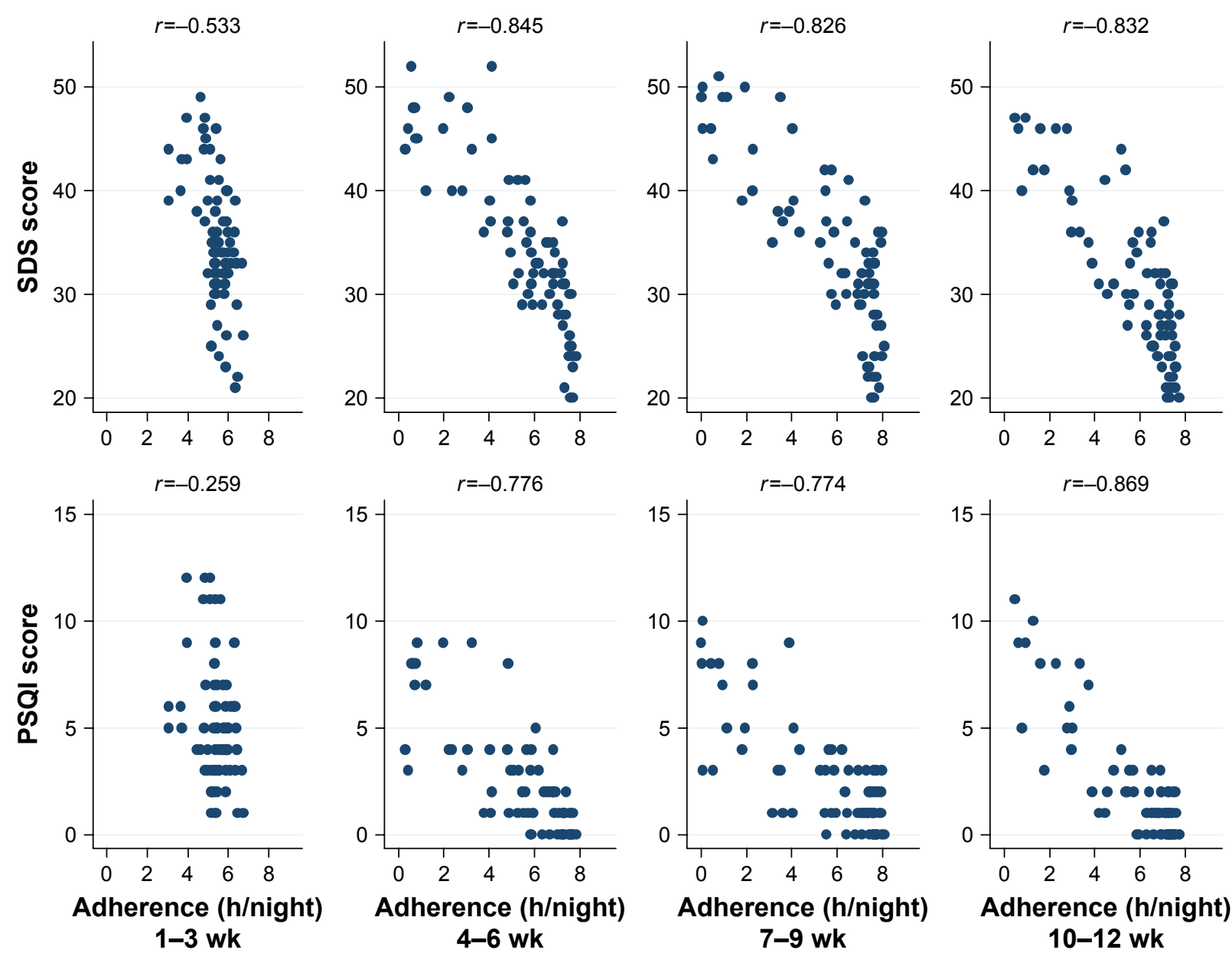

Figure 2 The crude relationship between adherence and SDS and PSQI scores in each 3-week period.

Notes: Each point represents one study subject. SDS, Zung's Self-Rating Depression Scale (20-80, higher score indicated more depressed) and PSQI, The Pittsburgh Sleep Quality Index (0-2I, higher score indicated lower sleep quality).

Abbreviations: h, hour; wk, weeks.

models were age, sex, BMI, AHI, adherence levels, therapy phases and the interaction between adherence level and therapy phase. Variables not showing significant contribution to the fit of the model were removed. To obtain an adjusted relationship between adherence range and the change in score from baseline for each scale, the initial influencing continuous variables, age, BMI and $\mathrm{AHI}$ were managed as the distance from mean, for example, age cen $=$ age - mean of age.

Table 2 Distributions of adherence levels at each therapy phase ( $N=76$ in each therapy phase)

\begin{tabular}{|c|c|c|c|c|c|}
\hline \multirow[t]{2}{*}{ Adherence } & \multicolumn{5}{|c|}{ Therapy phases (week) } \\
\hline & $\begin{array}{l}0 \\
\text { n (\%) }\end{array}$ & $\begin{array}{l}\mathrm{I}-3 \\
\mathrm{n}(\%)\end{array}$ & $\begin{array}{l}4-6 \\
\text { n (\%) }\end{array}$ & $\begin{array}{l}\text { 7-9 } \\
\text { n (\%) }\end{array}$ & $\begin{array}{l}10-12 \\
\text { n (\%) }\end{array}$ \\
\hline \multicolumn{6}{|c|}{ Adherence range } \\
\hline 0 hour & $76(100)$ & - & - & - & - \\
\hline$<4$ hours & - & $6(7.9)$ & $15(19.7)$ & $18(23.7)$ & $15(19.7)$ \\
\hline 4-6 hours & - & $57(75.0)$ & $23(30.3)$ & $12(15.8)$ & $14(18.4)$ \\
\hline$\geq 6$ hours & - & $13(17.1)$ & $38(50.0)$ & $46(60.5)$ & $47(61.8)$ \\
\hline
\end{tabular}

\section{ESS score}

As shown in Table 4, after model refinement, adherence level, therapy phase and BMI remained. The final model shows that nCPAP therapy could effectively reduce ESS score even at $<4$ hours adherence level; and with the increase in adherence levels, further reduction in ESS score could be achieved.

Figure 3A shows the adjusted relationship between the change from baseline of ESS score and adherence levels at each 3-week period. Controlling for BMI, the ESS scores decreased slightly with the increase in adherence, and this relationship was stable throughout the 4th to 12 th week of therapy.

\section{FSS score}

The final mixed-effects model showed that FSS score was influenced by adherence range and therapy phase. Unlike the ESS score, the lowest adherence range showed a nonsignificant effect on decreasing FSS score, but with the increase in adherence ranges, the FSS score improved significantly. Also 
Table 3 Distributions of ESS, FSS, SDS and PSQI scores across adherence levels through I2-week nCPAP therapy

\begin{tabular}{|c|c|c|c|c|c|}
\hline \multirow[t]{3}{*}{ Items } & \multicolumn{4}{|c|}{ Adherence levels } & \multirow[t]{3}{*}{$P *$-value } \\
\hline & 0 hour & $<\mathbf{4}$ hours & 4-6 hours & $\geq 6$ hours & \\
\hline & Mean (SD) & Mean (SD) & Mean (SD) & Mean (SD) & \\
\hline \multicolumn{6}{|l|}{ ESS } \\
\hline 0 & $12.66(4.92)$ & & & & \\
\hline I-3 weeks & & $5.17(1.72)$ & $5.96(2.54)$ & $5.69(2.25)$ & 0.725 \\
\hline 4-6 weeks & & $8.13(2.72)^{\mathrm{a}}$ & $6.96(2.40)^{\mathrm{a}}$ & $5.45(2.73)^{\mathrm{b}}$ & 0.003 \\
\hline 7-9 weeks & & $8.44(2.68)^{\mathrm{a}}$ & $6.75(2.99)^{a, b}$ & $5.98(2.76)^{b}$ & 0.009 \\
\hline $10-12$ weeks & & $8.93(2.60)^{\mathrm{a}}$ & $6.43(3.08)^{\mathrm{b}}$ & $5.26(2.59)^{\mathrm{b}}$ & $<0.001$ \\
\hline$P^{\#}$ & & 0.126 & 0.078 & 0.498 & \\
\hline \multicolumn{6}{|l|}{ FSS } \\
\hline 0 & $44.39(11.60)$ & & & & \\
\hline I-3 weeks & & A,B39.67 (I 4.40$)$ & A37.93 (I3.40) & A38.54 (9.67) & 0.946 \\
\hline 4-6 weeks & & ${ }^{A} 51.67(11.36)^{a}$ & ${ }^{\mathrm{A}} 41.96(1 \mathrm{I} .95)^{\mathrm{b}}$ & $\mathrm{A}, \mathrm{B} 30.32(\mathrm{II} .67)^{\mathrm{C}}$ & $<0.00 \mathrm{I}$ \\
\hline 7-9 weeks & & A50.39 (I3.14)a & ${ }^{A} 46.08(11.52)^{a}$ & ${ }^{B} 29.4 \mathrm{I}(12.52)^{\mathrm{b}}$ & $<0.001$ \\
\hline $10-12$ weeks & & ${ }^{\mathrm{B}} 45.33(\mathrm{II} .8 \mathrm{I})^{\mathrm{a}}$ & В35.86 (I I.43) & ${ }^{c} 26.55(11.56)^{c}$ & $<0.00 \mathrm{I}$ \\
\hline$P \#$ & & $<0.001$ & $<0.001$ & $<0.001$ & \\
\hline \multicolumn{6}{|l|}{ SDS } \\
\hline 0 & 37.39 (5.17) & & & & \\
\hline I-3 weeks & & A42.67 (2.88) & A34.98 (5.9I) & A3I. $23(5.33)^{c}$ & 0.001 \\
\hline 4-6 weeks & & A44.73 (4.23) & A35.96 (5.67) & ${ }^{B} 29.29(4.36)^{\text {с }}$ & $<0.001$ \\
\hline 7-9 weeks & & A444.56 (5.38) & A37.08 $(5.03)^{\mathrm{b}}$ & ${ }^{B} 29.54(5.14)^{\mathrm{C}}$ & $<0.00 \mathrm{I}$ \\
\hline $10-12$ weeks & & B $41.40(4.84)^{a}$ & B33.79 $(5.25)^{\mathrm{b}}$ & ${ }^{c} 26.57(4.30)^{c}$ & $<0.00 \mathrm{I}$ \\
\hline$P^{\#}$ & & $<0.00 \mathrm{I}$ & $<0.00 \mathrm{I}$ & $<0.001$ & \\
\hline \multicolumn{6}{|l|}{ PSQI } \\
\hline 0 & $5.25(2.58)$ & & & & \\
\hline I-3 weeks & & A7.17 (2.79) & A5.00 (2.79) & A4.3I (2.2I) & 0.104 \\
\hline 4-6 weeks & & ${ }^{B} 5.87(2.67)^{a}$ & ${ }^{\mathrm{B}} 2.30(1.77)^{\mathrm{b}}$ & ${ }^{\mathrm{B}} 1.05(1.18)^{\mathrm{C}}$ & $<0.001$ \\
\hline 7-9 weeks & & ${ }^{B} 5.67(2.89)^{a}$ & ${ }^{\mathrm{B}} 2.50(\mathrm{I} .62)^{\mathrm{b}}$ & ${ }^{\mathrm{B}} \mathrm{I} .22(1.07)^{\mathrm{c}}$ & $<0.001$ \\
\hline $10-12$ weeks & & ${ }^{B} 6.67(2.64)^{\mathrm{a}}$ & ${ }^{\mathrm{B}} 2.00(1.18)^{\mathrm{b}}$ & ${ }^{\mathrm{B}} 0.83(0.84)^{\mathrm{c}}$ & $<0.001$ \\
\hline$P^{\#}$ & & $<0.001$ & $<0.001$ & $<0.001$ & \\
\hline
\end{tabular}

Notes: In the row with $P<0.05$, the values that do not share a common superscript (right side small letters, such as a, b, c) differ significantly from one another ( $<<0.05$, two-sample $t$-test). In the column with $P<0.05$, the values that do not share a common superscript (left side capital letters, such as $A$, B, $C$ ) differ significantly from one another ( $P<0.05$, mixed-effects regression). *, One-way ANOVA analysis; *, mixed-effect regression. ESS, Epworth Sleepiness Scale (0-24, higher score indicated sleepier); FSS, Fatigue Severity Scale (9-63, higher score indicated more fatigue); SDS, Zung's Self-Rating Depression Scale (20-80, higher score indicated more depressed); PSQI, The Pittsburgh Sleep Quality Index (0-2I, higher score indicated lower sleep quality).

Abbreviations: nCPAP, nasal continuous positive airway pressure; ANOVA, analysis of variance; SD, standard deviation.

different from the trend of ESS score in therapy phases, the FSS score decreased significantly at 10- to 12-week therapy phase, compared with the first 3-week therapy phase (Table 4). No other factors were identified as being significantly associated with FSS score.

Figure 3B shows the relationship between the change in FSS score from baseline and adherence range, which was stratified by therapy phases. With the increase in adherence range, the FSS score decreased. The most significant improvement was at 10- to 12-week therapy.

\section{SDS score}

Table 4 shows the result of refined model. SDS score was influenced by adherence range, therapy phase, age and sex. The nCPAP therapy could significantly reduce SDS score with $\geq 4$ hours adherence and the effects enhanced with the increasing adherence. The multivariate model also revealed a significantly reduced SDS score at the last therapy phase. Female sex and increasing age correlated with higher SDS scores.

Figure $3 \mathrm{C}$ shows the adjusted relationship between adherence range and the change in SDS scores from baseline in 4 therapy phases in males adjusted for age. The lines in the figure of females would be parallel $\sim 3.6$ units higher (Table 4) compared with the figure of males. The SDS scores slightly decreased with the increasing adherence, but a significant drop from baseline occurred at the last therapy phase (10-12 weeks).

\section{PSQI score}

Table 4 shows that adherence range, therapy phase and sex influenced PSQI score. Different from ESS scores, PSQI 
Table 4 Mixed-effects models of influencing factors of ESS, FSS, SDS and PSQI scores during 3-month nCPAP therapy

\begin{tabular}{|c|c|c|c|c|c|c|c|c|}
\hline Variables & ESS & $P$-value & FSS & $P$-value & SDS & $P$-value & PSQI & $P$-value \\
\hline Adherence range & & $<0.001$ & & $<0.001$ & & $<0.001$ & & 0.023 \\
\hline 0 hour & $0^{\mathrm{a}}$ & & $0^{\mathrm{a}}$ & & $0^{\mathrm{a}}$ & & $0^{\mathrm{a}}$ & \\
\hline$<4$ hours & $-4.49(-5.62,-3.36)^{\mathrm{b}}$ & & $0.70(-1.18,2.59)^{a}$ & & $\mathrm{I} .35(0.4 \mathrm{I}, 2.29)^{\mathrm{b}}$ & & $0.30(-0.24,0.83)^{a}$ & \\
\hline 4-6 hours & $-6.69(-7.40,-5.99)^{c}$ & & $-6.02(-7.14,-4.91)^{b}$ & & $-2.40(-2.95,-1.85)^{\mathrm{c}}$ & & $-0.20(-0.52,-0.12)^{\mathrm{b}}$ & \\
\hline$\geq 6$ hours & $-8.35(-9.26,-7.44)^{d}$ & & $-10.30(-11.78,-8.83)^{c}$ & & $-4.42(-5.15,-3.68)^{d}$ & & $-0.40(-0.82,-0.02)^{\mathrm{b}}$ & \\
\hline Therapy phases & & 0.030 & & $<0.001$ & & $<0.001$ & & $<0.001$ \\
\hline I-3 weeks & $0^{\mathrm{a}}$ & & $0^{\mathrm{a}}$ & & $0^{a}$ & & $0^{\mathrm{a}}$ & \\
\hline 4-6 weeks & $0.86(0.1 \mathrm{I}, 1.62)^{\mathrm{b}}$ & & $0.49(-0.71,1.69)^{\mathrm{a}}$ & & $-0.37(-0.97,0.22)^{\mathrm{a}}$ & & $-2.67(-3.0 \mathrm{I},-2.32)^{\mathrm{b}}$ & \\
\hline 7-9 weeks & $1.20(0.40,2.00)^{\mathrm{b}}$ & & $-0.36(-1.65,0.93)^{\mathrm{a}}$ & & $-0.37(-1.01,0.27)^{\mathrm{a}}$ & & $-2.57(-2.94,-2.21)^{b}$ & \\
\hline $10-12$ weeks & $0.84(0.04,1.63)^{b}$ & & $-5.08(-6.35,-3.8 \mathrm{I})^{\mathrm{b}}$ & & $-3.66(-4.29,-3.03)^{\mathrm{b}}$ & & $-2.83(-3.19,-2.47)^{b}$ & \\
\hline $\mathrm{Age}_{\text {cen, }}$, years & NS & & NS & & $0.12(0.04,0.21)$ & 0.003 & NS & \\
\hline Sex & & & & & & 0.013 & & $<0.001$ \\
\hline Female & NS & & NS & & $3.57(0.77,6.38)$ & & $2.21(1.07,3.35)$ & \\
\hline $\mathrm{BMI}_{\mathrm{cen}}, \mathrm{kg} / \mathrm{m}^{2}$ & $0.36(0.20,0.52)$ & $<0.00$ I & NS & & NS & & NS & \\
\hline Constant & $12.65(11.97,13.34)$ & $<0.001$ & $44.40(41.59,47.20)$ & $<0.001$ & $36.55(35.18,37.92)$ & $<0.001$ & $4.73(4.14,5.31)$ & $<0.001$ \\
\hline
\end{tabular}

Notes: The potential influence predictors on ESS, FSS, SDS and PSQI initially included in mixed-effects models were age, sex, BMI, AHI, adherence levels, therapy phases and the interaction between adherence level and therapy phase. For these variables with $>2$ levels, coefficients not sharing a common superscript (a,b,c, or $\mathrm{d}$ ) differ significantly $\left(P<0.05\right.$, Wald test). Age ${ }_{\text {cen }}$, deviation of age from mean; BMI ${ }_{\text {cen }}$ deviation of BMI from mean. ESS, Epworth Sleepiness Scale (0-24, higher score indicated sleepier); FSS, Fatigue Severity Scale (9-63, higher score indicated more fatigue); SDS, Zung's Self-Rating Depression Scale (20-80, higher score indicated more depressed); PSQI, The Pittsburgh Sleep Quality Index (0-21, higher score indicated lower sleep quality).

Abbreviations: nCPAP, nasal continuous positive airway pressure; NS, nonsignificant; BMI, body mass index; AHI, apnea/hypopnea index.

A

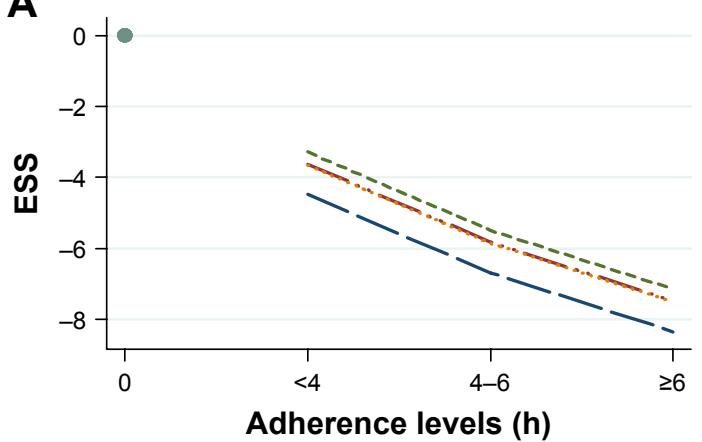

C

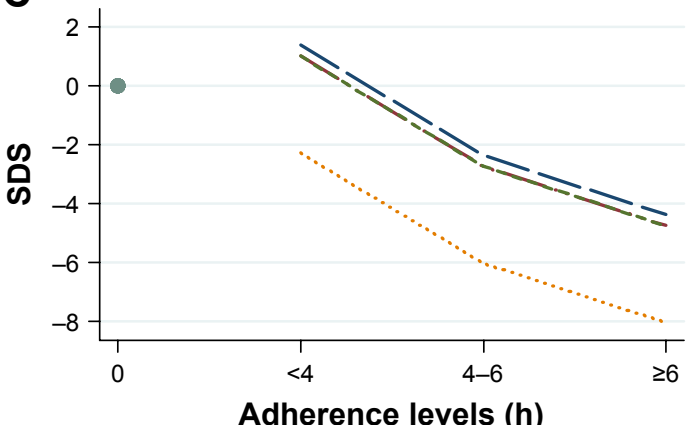

B

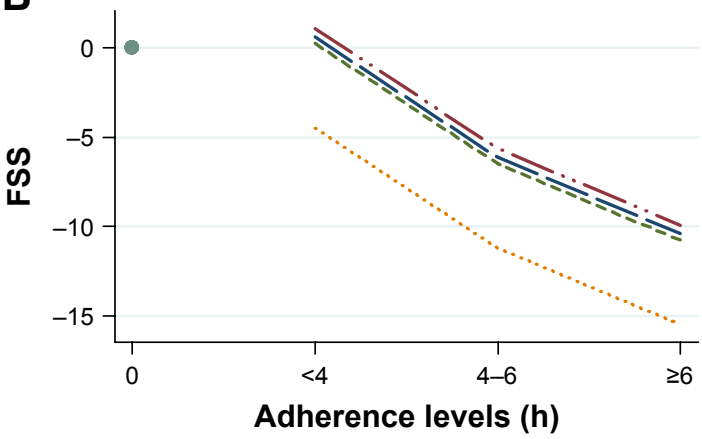

D

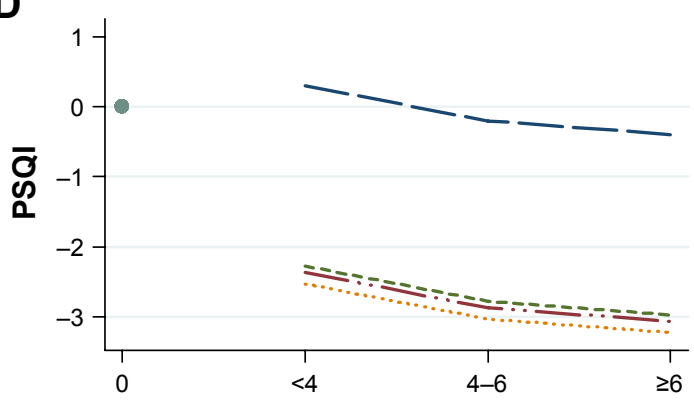

Adherence levels ( $h$ )

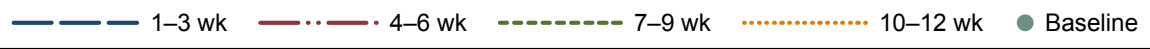

Figure 3 Adjusted relationships between adherence range and the changes in ESS, FSS, SDS and PQSI scores from baseline within each 3-week period during first 3 months of nCPAP therapy.

Notes: (A) Adjusted relationship between the change in ESS score from baseline and adherence range within each 3-week period during first 3 months of nCPAP therapy; the mean of adjusted ESS score at baseline was 12.66. (B) Adjusted relationship between the change in FSS score from baseline and adherence range within each 3-week period during first 3 months of nCPAP therapy; the mean of adjusted FSS score at baseline was 44.40. (C) Adjusted relationship between the change in SDS score from baseline and adherence range within each 3 -week period during first 3 months of nCPAP therapy among males; the mean of adjusted SDS score at baseline was 36.55 . (D) Adjusted relationship between the change in PSQI score from baseline and adherence range within each 3-week period during first 3 months of nCPAP therapy among males; the mean of adjusted PSQI score at baseline was 4.73. ESS, Epworth Sleepiness Scale (0-24, higher score indicated sleepier); FSS, Fatigue Severity Scale (9-63, higher score indicated more fatigue); SDS, Zung's Self-Rating Depression Scale (20-80, higher score indicated more depressed); PSQI, The Pittsburgh Sleep Quality Index (0-2I, higher score indicated lower sleep quality).

Abbreviations: nCPAP, nasal continuous positive airway pressure; h, hours; wk, weeks. 
score did not show a significant decrease unless the adherence was $\geq 4$ hours per night. Controlling for adherence range, therapy phase was an independent factor that significantly decreased PSQI score compared with the first 3-week therapy. Worse sleep quality occurred in females.

Figure 3D shows the adjusted relationship between adherence range and the change in PSQI score from baseline at each therapy phase in males. The lines in the figure of females would be parallel 2.2 units higher (Table 4) compared with the figure of males. The extent of reduction in PSQI score at 4-6 hours adherence was greater than that at $<4$ hours; however, adherence of $\geq 6$ hours did not show a further benefit. The effect of therapy phase was significantly different from first to second 3-week period. No more benefit was seen after the 6 th week.

\section{Discussion}

In this study, the mixed-effect models revealed the independent effect of adherence range, $<4$ hours, 4-6 hours and $\geq 6$ hours per night using nCPAP, on day- and nighttime symptoms. Even $<4$ hours adherence could improve ESS score significantly. The significant improvement in FSS and SDS scores required a higher adherence ( $\geq 4$ hours) than that of ESS score. With increasing adherence, more benefit could be achieved in ESS, FSS and SDS. Compared with the effect of adherence on daytime symptoms, the improvement in nighttime symptom, in terms of PSQI score, was mainly influenced by the progress of therapy phases.

The effect of adherence to nCPAP therapy on daytime sleepiness has been studied, especially the relationship between adherence levels and normalization of ESS score..$^{10,11,15,22-25}$ Two important studies had been reported by Weaver et $\mathrm{al}^{11}$ and Antic et al, ${ }^{10}$ which demonstrated that using CPAP $\geq 4$ hours per night could normalize 60\%-70\% of EDS patients' ESS score after 3-month therapy. Compared with previous studies ${ }^{10,11}$ that categorized ESS score into normal or abnormal, in this study we focused on the relationship between average adherence at each 3-week period and the exact ESS score measured at the end of each 3-week period. Despite the different types of ESS data between previous studies $^{10,11}$ and the current study, a similar relationship was found that with the increase in adherence, the patients obtained significant further improvement in ESS score. Moreover, the interaction between adherence ranges and therapy phases was nonsignificant in this study, which suggested that this relationship was stable through the therapy phase.

In this study, after the first therapy phase, the mean of ESS score among OSAS patients (mean [SD], 5.85 [2.43]) was close to the result of local 3085 university teachers and staff population (5.14 [3.78]), ${ }^{26}$ which indicated that the ESS score of OSAS patients achieved an average level of community population. After that, patients had to take therapy continually to maintain this therapy result in the subsequent phases. To maintain the therapy result of first 3 weeks, patients need to retain the adherence to nCPAP therapy at least $>4$ hours per night to overcome the increasing trend of ESS caused by OSAS; this result also supported the definition of adherence in previous studies..$^{10,11,15,22-25}$

Fatigue as a frequent complaint in OSAS patients ${ }^{7,27-32}$ has been studied in the recent decade..$^{30,33-35}$ In this study, we found that the most significant improvement in FSS score was at 10- to 12-week therapy phase, which was in agreement with the previous study. ${ }^{13}$ In contrast, ESS score improved most notably at the 3 rd week therapy. If fatigue was mostly caused by unrefreshing fragmented sleep, the FSS score and ESS score might be expected to have similar timing of improvement. Therefore, the causation of fatigue and daytime sleepiness might be not identical in OSAS patients. However, we found that the most improvement in SDS and FSS scores was at the same therapy phase, which was between 9th and 12 th week of therapy. This is consistent with the report that depression was one cause of fatigue. ${ }^{36}$ Moreover, Mills et al ${ }^{37}$ reported that the collapse of upper airway could increase not only local inflammation but also systemic inflammation. Fatigue as one of the results of inflammation, ${ }^{38}$ the FSS score also might be influenced by the local and/or systemic inflammation; and a systemic review indicated that the significant improvement in systematic inflammation needed at least 3 months adequate ( $\geq 4$ hours per night) CPAP therapy, ${ }^{39}$ which was potentially consistent with the most significant improvement phase of this study. Therefore, the improvement in fatigue in OSAS patient might require a better adherence and a longer term than daytime sleepiness.

The effect of CPAP therapy on improvement in depression has been studied. ${ }^{8,40-43}$ However, the effect of CPAP on depression remains controversial. ${ }^{8}$ In this study, after controlling for age and sex and therapy phase, the adherence level was an independent influencing factor of SDS score, and more improvement in SDS score could be achieved by an increase in adherence. Female sex and aging were also reported as risk factors of depression among OSAS patients by previous study. ${ }^{16}$ This result might hint the importance of controlling the effect of females and elderly patients in related studies. Another confusion of the effectiveness of nCPAP on SDS was that $<4$ hours adherence could significantly increase SDS score in the first 9 weeks. One potential 
explanation was that some OSAS patients' depression status might not be caused by OSAS, but it might be primary depression coexisting with OSAS. It was also reported that depression was a risk factor of poor adherence to CPAP, ${ }^{14}$ so that primary depression status might reduce the patients' level of adherence, and these primary depression patients would obtain limited benefit of nCPAP therapy. Hence, the effectiveness of CPAP therapy on depression status might be underestimated when pooling primary and nonprimary depression patients together.

As mentioned earlier, we observed that the most improvement in SDS score was at 10- to 12-week therapy phase. Because of the few studies designed as multiple measurements of depression with a 3-week interval, we could not compare the effect of therapy phase within 1 study. Nevertheless, some previous studies might support our finding. According to the literature, ${ }^{44} 2$ - to 3 -week therapy might not show a specific therapeutic effect on mood symptoms; ${ }^{40,44}$ 4- to 6-week therapy might produce a significant improvement in depression score under the condition of high adherence of therapy ( $\geq 6$ hours per night); ${ }^{41} 8$ weeks ${ }^{42,45}$ or 3 months ${ }^{43}$ could significantly improve depression score with appropriate adherence ( $>4$ hours). Therefore, the timing of evaluation was also important for the assessment of effectiveness of CPAP therapy on depression status.

The significant effectiveness of CPAP therapy on objective sleep quality such as rapid eye movement sleep and arousal has been proved by previous studies, ${ }^{5,46-48}$ however, the effectiveness on subjective sleep quality has not often been reported. ${ }^{34,49}$ Previous studies reported that subjective sleep quality of newly diagnosed OSAS patients significantly improved after nCPAP therapy. ${ }^{34,49}$ This finding was similar to the result of univariate analysis in this study. However, the result of mixedeffect model showed a limited effect of adherence level on the improvements in PSQI score, and the main improvement was caused by the progress of therapy phases. CPAP could significantly improve objective sleep quality; however, using a CPAP device during sleep might lead patients to feel uncomfortable and thus decrease subjective sleep quality despite the correction of apnea. The benefit of improvement in objective sleep quality might improve subjective sleep quality only if the patient was accustomed the sleeping with the CPAP device. This might be partially supported by the results of the above-mentioned 2 long-term studies. ${ }^{34,49}$

\section{Conclusion}

The nCPAP therapy could effectively improve daytime sleepiness in first 3-week therapy phase even the adherence level $<4$ hours per night. However, the significant improvements in fatigue and depression required a better adherence and longer term. The changes in sleep quality mostly depended on whether the patient was accustomed the sleeping with the nCPAP device. In addition, the mixed-effects models showed the nonsignificance of interaction between adherence level and therapy phase in day- and nighttime symptoms, which suggested that this relationship was stable through the therapy phase.

\section{Limitations of this study}

The results of this study were based on a 12-week follow-up, which might limit the ability to generalize from our results to long-term effect of adherence on the abovementioned clinical symptoms. This study repeated 5 times the measurements among 76 OSAS patients. According to the study design, the sample size could meet the sufficient power of univariate analysis; however, for the multiple analysis, it was related to the lack of statistical power.

\section{Acknowledgments}

This study was supported by the Yunnan Provincial young and middle-aged academic leaders reserve talent program (2011CZ048), National Natural Science Foundation of China (81460017 and 81660019) and the scientific research program of Yunnan Education Department (2017zzx198).

\section{Disclosure}

The authors report no conflicts of interests in this work.

\section{References}

1. Guilleminault C, Tilkian A, Dement WC. The sleep apnea syndromes. Annu Rev Med. 1976;27:465-484.

2. American Academy of Sleep Medicine. International Classification of Sleep Disorders, Revised: Diagnostic and Coding Manual. Chicago, IL: American Academy of Sleep Medicine; 2001.

3. Roux F, Hilbert J. Continuous positive airway pressure: new generations Clin Chest Med. 2003;24(2):315-342.

4. Weaver TE. Adherence to continuous positive airway pressure treatment and functional status in adult obstructive sleep apnea. In: Pack AI, editor. Sleep Apnea Pathogenesis Diagnosis and Treatment. New York, NY: Marcel Dekker; 2002:523:554.

5. McArdle N, Douglas NJ. Effect of continuous positive airway pressure on sleep architecture in the sleep apnea-hypopnea syndrome: a randomized controlled trial. Am J Respir Crit Care Med. 2001;164(8 pt 1): 1459-1463.

6. Saunamaki T, Himanen SL, Polo O, Jehkonen M. Executive dysfunction in patients with obstructive sleep apnea syndrome. Eur Neurol. 2009; 62(4):237-242.

7. Tomfohr LM, Ancoli-Israel S, Loredo JS, Dimsdale JE. Effects of continuous positive airway pressure on fatigue and sleepiness in patients with obstructive sleep apnea: data from a randomized controlled trial. Sleep. 2011;34(1):121-126.

8. Povitz M, Bolo CE, Heitman SJ, Tsai WH, Wang J, James MT. Effect of treatment of obstructive sleep apnea on depressive symptoms: systematic review and meta-analysis. PLoS Med. 2014;11(11):e1001762. 
9. Wang Y, Geater AF, Chai Y, et al. Pre- and in-therapy predictive score models of adult OSAS patients with poor adherence pattern on nCPAP therapy. Patient Prefer Adherence. 2015;9:715-723.

10. Antic NA, Catcheside P, Buchan C, et al. The effect of CPAP in normalizing daytime sleepiness, quality of life, and neurocognitive function in patients with moderate to severe OSA. Sleep. 2011; 34(1):111-119.

11. Weaver TE, Maislin G, Dinges DF, et al. Relationship between hours of CPAP use and achieving normal levels of sleepiness and daily functioning. Sleep. 2007;30(6):711-719.

12. Woehrle H, Graml A, Weinreich G. Age- and gender-dependent adherence with continuous positive airway pressure therapy. Sleep Med.2011; 12(10):1034-1036.

13. Powell ED, Gay PC, Ojile JM, Litinski M, Malhotra A. A pilot study assessing adherence to auto-bilevel following a poor initial encounter with CPAP. J Clin Sleep Med. 2012;8(1):43-47.

14. Law M, Naughton M, Ho S, Roebuck T, Dabscheck E. Depression may reduce adherence during CPAP titration trial. J Clin Sleep Med. 2014; 10(2):163-169.

15. Sin DD, Mayers I, Man GC, Pawluk L. Long-term compliance rates to continuous positive airway pressure in obstructive sleep apnea: a population-based study. Chest. 2002;121(2):430-435.

16. Gagnadoux F, Le Vaillant M, Goupil F, et al. Depressive symptoms before and after long-term CPAP therapy in patients with sleep apnea. Chest. 2014;145(5):1025-1031.

17. Chinese Society of Respiratory Diseases. Obstructive sleep apnea/ hypopnea diagnosis guideline. Chin J Tuberc Respir Dis. 2012;35:9-12. Chinese.

18. Johns MW. A new method for measuring daytime sleepiness: the Epworth sleepiness scale. Sleep. 1991;14(6):540-545.

19. Krupp LB, LaRocca NG, Muir-Nash J, Steinberg AD. The fatigue severity scale. Application to patients with multiple sclerosis and systemic lupus erythematosus. Arch Neurol. 1989;46(10):1121-1123.

20. Zung WW. A Self-Rating Depression Scale. Arch Gen Psychiatry. 1965; 12:63-70.

21. Buysse DJ, Reynolds CF 3rd, Monk TH, Berman SR, Kupfer DJ. The Pittsburgh Sleep Quality Index: a new instrument for psychiatric practice and research. Psychiatry Res. 1989;28(2):193-213.

22. Pepin JL, Viot-Blanc V, Escourrou P, et al. Prevalence of residual excessive sleepiness in CPAP-treated sleep apnoea patients: the French multicentre study. Eur Respir J. 2009;33(5):1062-1067.

23. Santamaria J, Iranzo A, Ma Montserrat J, de Pablo J. Persistent sleepiness in CPAP treated obstructive sleep apnea patients: evaluation and treatment. Sleep Med Rev. 2007;11(3):195-207.

24. Muñoz A, Mayoralas LR, Barbé F, et al. Long-term effects of CPAP on daytime functioning in patients with sleep apnoea syndrome. Eur Respir J. 2000;15(4):676-681.

25. Sforza E, Krieger J. Daytime sleepiness after long-term continuous positive airway pressure (CPAP) treatment in obstructive sleep apnea syndrome. J Neurol Sci. 1992;110(1-2):21-26.

26. Wang Y, Sun X, Cao Y, Li Y. Prevalence of excessive daytime sleepiness and associated factors in 3085 university teachers and staffs in Kunming. J Kunming Med Univ. 2013;34(8):13-18. Chinese.

27. Le Bon O, Fischler B, Hoffmann G, et al. How significant are primary sleep disorders and sleepiness in the chronic fatigue syndrome? Sleep Res Online. 2000;3(2):43-48.

28. Chervin RD. Sleepiness, fatigue, tiredness, and lack of energy in obstructive sleep apnea. Chest. 2000;118(2):372-379.

29. Ohayon MM, Shapiro CM. Sleep and fatigue. Semin Clin Neuropsychiatry. 2000;5(1):56-57.

30. Dias RA, Hardin KA, Rose H, Agius MA, Apperson ML, Brass SD. Sleepiness, fatigue, and risk of obstructive sleep apnea using the STOPBANG questionnaire in multiple sclerosis: a pilot study. Sleep Breath. 2012;16(4):1255-1265.
31. Jackson ML, Stough C, Howard ME, Spong J, Downey LA, Thompson B. The contribution of fatigue and sleepiness to depression in patients attending the sleep laboratory for evaluation of obstructive sleep apnea. Sleep Breath. 2011;15(3):439-445.

32. Bardwell WA, Ancoli-Israel S, Dimsdale JE. Comparison of the effects of depressive symptoms and apnea severity on fatigue in patients with obstructive sleep apnea: a replication study.J Affect Disord. 2007;97(1-3): 181-186.

33. Chotinaiwattarakul W, O'Brien LM, Fan L, Chervin RD. Fatigue, tiredness, and lack of energy improve with treatment for OSA. J Clin Sleep Med. 2009;5(3):222-227.

34. Bartlett D, Wong K, Richards D, et al. Increasing adherence to obstructive sleep apnea treatment with a group social cognitive therapy treatment intervention: a randomized trial. Sleep. 2013;36(11):1647-1654.

35. Veauthier C, Gaede G, Radbruch H, Gottschalk S, Wernecke KD, Paul F. Treatment of sleep disorders may improve fatigue in multiple sclerosis. Clin Neurol Neurosurg. 2013;115(9):1826-1830.

36. Saltiel PF, Silvershein DI. Major depressive disorder: mechanism-based prescribing for personalized medicine. Neuropsychiatr Dis Treat. 2015; 11:875-888.

37. Mills PJ, Dimsdale JE. Sleep apnea: a model for studying cytokines, sleep, and sleep disruption. Brain Behav Immun. 2004;18(4):298-303.

38. Dantzer R, Capuron L, Irwin MR, et al. Identification and treatment of symptoms associated with inflammation in medically ill patients. Psychoneuroendocrinology. 2008;33(1):18-29.

39. Xie X, Pan L, Ren D, Du C, Guo Y. Effects of continuous positive airway pressure therapy on systemic inflammation in obstructive sleep apnea: a meta-analysis. Sleep Med. 2013;14(11):1139-1150.

40. Haensel A, Norman D, Natarajan L, Bardwell WA, Ancoli-Israel S, Dimsdale JE. Effect of a 2 week CPAP treatment on mood states in patients with obstructive sleep apnea: a double-blind trial. Sleep Breath. 2007;11(4):239-244.

41. Schwartz DJ, Kohler WC, Karatinos G. Symptoms of depression in individuals with obstructive sleep apnea may be amenable to treatment with continuous positive airway pressure. Chest. 2005;128(3):1304-1309.

42. Kawahara S, Akashiba T, Akahoshi T, Horie T. Nasal CPAP improves the quality of life and lessens the depressive symptoms in patients with obstructive sleep apnea syndrome. Intern Med. 2005;44(5): 422-427.

43. Edwards C, Mukherjee S, Simpson L, Palmer LJ, Almeida OP, Hillman DR. Depressive symptoms before and after treatment of obstructive sleep apnea in men and women. J Clin Sleep Med. 2015;11(9): 1029-1038.

44. Lee IS, Bardwell W, Ancoli-Israel S, Loredo JS, Dimsdale JE. Effect of three weeks of continuous positive airway pressure treatment on mood in patients with obstructive sleep apnoea: a randomized placebocontrolled study. Sleep Med. 2012;13(2):161-166.

45. Habukawa M, Uchimura N, Kakuma T, et al. Effect of CPAP treatment on residual depressive symptoms in patients with major depression and coexisting sleep apnea: Contribution of daytime sleepiness to residual depressive symptoms. Sleep Med. 2010;11(6):552-557.

46. Loredo JS, Ancoli-Israel S, Dimsdale JE. Effect of continuous positive airway pressure vs placebo continuous positive airway pressure on sleep quality in obstructive sleep apnea. Chest. 1999;116(6):1545-1549.

47. Loredo JS, Ancoli-Israel S, Kim EJ, Lim WJ, Dimsdale JE. Effect of continuous positive airway pressure versus supplemental oxygen on sleep quality in obstructive sleep apnea: a placebo-CPAP-controlled study. Sleep. 2006;29(4):564-571.

48. Hsu CC, Wu JH, Chiu HC, Lin CM. Evaluating the sleep quality of obstructive sleep apnea patients after continuous positive airway pressure treatment. Comput Biol Med. 2013;43(7):870-878.

49. Mermigkis C, Bouloukaki I, Antoniou K, et al. Obstructive sleep apnea should be treated in patients with idiopathic pulmonary fibrosis. Sleep Breath. 2015;19(1):385-391 
Patient Preference and Adherence

Dovepress

\section{Publish your work in this journal}

Patient Preference and Adherence is an international, peer-reviewed, open access journal that focuses on the growing importance of patient preference and adherence throughout the therapeutic continuum. Patient satisfaction, acceptability, quality of life, compliance, persistence and their role in developing new therapeutic modalities and compounds to optimize

Submit your manuscript here: http://www.dovepress.com/patient-preference-and-adherence-journ clinical outcomes for existing disease states are major areas of interest for the journal. This journal has been accepted for indexing on PubMed Central The manuscript management system is completely online and includes a very quick and fair peer-review system, which is all easy to use. Visit http://www. dovepress.com/testimonials.php to read real quotes from published authors. 